\title{
Non-clinic blood pressure measurement - more light, less darkness
}

\author{
Vinay Kapur \\ Associate Professor, Department of Medicine,Dr H S Judge Institute Of Dental sciences, Panjab University, Chandigarh
}

Submitted: 13-09-2018

Revised: 24-10-2018

Published: 01-01-2019

A B STRACT

Non-clinic blood pressure measurements are very important in confirming diagnosis of hypertension and they give us an idea of associated cardiovascular risk more precisely than clinic blood pressure measurements. It can detect masked hypertension and white coat hypertension and ambulatory blood pressure monitoring (ABPM) especially can monitor night-time blood pressure diagnosing individuals with nocturnal hypertension. Masked hypertension and nocturnal hypertension are strongly related with target organ damage along with enhanced morbidity and mortality due to cardiovascular causes.ABPM can also measure early morning rise of blood pressure, mean 24 hour blood pressure, diurnal variation as well as average real variability. Home blood pressure monitoring by patients themselves leads to their greater involvement in maintaining blood pressure records and in achieving treatment goals. The purpose of this review is to preferentially discuss role of non-clinic blood pressure monitoring in making accurate diagnosis and deciding treatment of hypertension that might help a great deal in reducing morbidity and mortality associated with it.

Access this article online

Website:

http://nepjol.info/index.php/AJMS DOI: 10.3126/ajms.v10i1.21077 E-ISSN: 2091-0576 P-ISSN: 2467-9100

Key words: Non-clinic blood pressure monitoring; White-coat hypertension; Masked hypertension; Non-dippers; Reverse dippers; Chronotherapy

\section{INTRODUCTION}

Clinic blood pressure measurement is time-honoured method for evaluating and treating hypertension but this routine method is being questioned for some time as a large number of individuals have varied blood pressure when measured outside clinic, at workplace or at home. Non-clinic blood pressure measurements are becoming more important in diagnosis as well as management of hypertension. Non clinic blood pressure can be monitored by two methods - Home blood pressure monitoring andAmbulatory blood pressure monitoring (ABPM). These methods are better than clinic blood pressure values in assessment of cardiovascular morbidity and mortality. Blood pressure not only varies with the place where it is measured but also depends on the person measuring it, highest measurements being obtained by physician followed by nurse and patient himself. ${ }^{1,2}$ Home blood pressure monitoring is easy to access and inexpensive while ABPM is expensive and not easily available. Despite these limitations, ABPM is gold standard for monitoring 24 hour blood pressure as it is a stronger predictor of hypertension related outcomes than clinic blood pressure readings.

\section{HOME BLOOD PRESSURE MONITORING}

In this method, a family member or patient himself measures blood pressure either at home or at work place. Two readings are obtained, 1 minute apart and their average is considered. Blood pressure should be checked before taking drugs two times in the morning and before dinner in evening. ${ }^{3}$ Blood pressure readings on first day are not considered and average of remaining readings is taken. A structured blood pressure log obtained over one week gives valuable information regarding blood pressure control. Once patients reach clinical stability, this monitoring is repeated every 1 - 2 months to be sure that blood pressure is within normal limits. 
Frequent monitoring (4-6 times per day) can be done in patients with labile hypertension to assess degree of blood pressure variability. Patients who develop hypotensive symptoms also require blood pressure monitoring when action of drug is at peak or when symptoms appear. Most acceptable blood pressure levels at home are less than 130-135/80-85 mmHg.

\section{Indications}

i) Any patient with hypertension.

ii) To rule out white coat/masked hypertension.

iii) Evaluation of resistant hypertension and labile hypertension.

iv) Evaluation of medication related hypotensive symptoms.

\section{Ambulatory blood pressure monitoring}

A light weight, portable device is attached to arm cuff and it gives us data in form of semi continuous blood pressure recording. Blood pressure monitoring is usually done for 24 hours but in some patients like those on haemodialysis, it can be extended upto 48 hours during entire interdialytic phase. The cuff is programmed such that inflation frequency is once every $20-30$ minutes during day time and it decreases to once every $30-60$ minutes during sleep. Frequency of inflations can be increased for patients having severe autonomic dysfunction or those having labile hypertension. ${ }^{4}$

Test should be performed on a day which matches patient's daily routine including time at work and at home. Patient is asked to record time of waking up and retiring as well as taking of any vasoactive drugs and time of any stressful activity during the test. Test is usually well tolerated but sleep can be affected in $10 \%$ of patients. Sleep records should be discarded in such patients. Time spent in siestas during daytime should also be added to sleep period. ${ }^{4}$ An adequate monitoring should have minimum of twenty valid measurements during awake period and minimum seven during sleep. ${ }^{5}$

ABPM also gives information about blood pressure variability during 24 hours based on standard deviation of blood pressure recordings and helps in evaluation of marked blood pressure fluctuations. It also tells about mean systolic and diastolic blood pressure and pressure changes during sleep and wakefulness. Following are essential components to be analysed in ABPM tracing:-

a) 24 hour blood pressure - average of all blood pressure recordings during 24 hour period and normal is < 120/80 mmHg. ${ }^{5}$

b) Awake blood pressure - average of all blood pressure recordings during awake period and average of $<$ $135 / 85 \mathrm{mmHg}$ is considered normal. ${ }^{5}$ c) Sleep blood pressure-average of all blood pressure recordings during sleep including measurements during siesta. An average of $<120 / 70 \mathrm{mmHg}$ is considered normal. ${ }^{5}$

d) Night/day blood pressure ratio i.e. ratio of average blood pressure during sleep and awake period.(Mean daytime pressure - mean night time pressure) X 100/ mean daytime pressure is the formula to calculate fall in blood pressure during sleep.

Blood pressure falls during sleep by approximately $15 \%$ equivalent to ratio of 0.85 . Taking this ratio as basis, patients are categorised into dippers (ratio 0.8-0.89), nondippers (ratio 0.9-1.0), reverse dippers (ratio $>1.0$ ) and extreme dippers (ratio < 0.8 ). This can be simply expressed as dippers (fall in BP during sleep $\geq 10 \%$ ), non-dippers (fall $<10 \%$ ), extreme dippers $(\geq 20 \%)$ and reverse dippers/ risers $(\leq 0 \%){ }^{6}$

\section{Indications ${ }^{6}$}

i) Suspected white coat hypertension.

ii) Suspected masked hypertension.

iii) Evaluation of efficacy of treatment when

(a) Casual blood pressure is not controlled despite optimal drug treatment.

b) Casual blood pressure is controlled but target organ damage has progressed.

iv) Evaluation of hypotensive symptoms.

v) Assessment of blood pressure variability

vi) Assessment for nocturnal hypertension or dipping pattern in conditions like chronic kidney disease, diabetes mellitus and obstructive sleep apnea.

\section{Advantages ${ }^{6}$}

i) Multiple blood pressure readings for 24 hours including during daily routine activities and sleep.

ii) Assessment of circadian rhythm of blood pressure.

iii) Assessment of blood pressure overload and variability.

iv) Assessment of effect of antihypertensive drugs over 24 hours.

v) Possibly risk stratification of hypertensive patients.

\section{Limitations $^{6}$}

i) Mismatch between cuff size and arm circumference.

ii) Very high systolic blood pressures.

iii) Presence of movement disorders e.g. essential tremors, parkinsonism

iv) In rhythm disorders due to irregular pulse.

v) Presence of auscultatory gap during routine manual blood pressure measurement.

ABPM and assessment of blood pressure behaviour along with different hypertension diagnoses 
Main objective of ABPM is to decide whether to treat a patient or not on basis of 24 hour blood pressure readings as two types of errors can occur if therapy is started based on casual blood pressure readings. First, if casual blood pressure overestimates real pressure, therapy can be started when it is not required and second, if it underestimates real value, therapy is withheld and can lead to adverse consequences of hypertension.

Depending on blood pressure values obtained during clinic measurements, four diagnoses can be identified: Normotension, hypertension, white coat hypertension and masked hypertension. ${ }^{6}$ Normotension is defined as clinic blood pressure $(<140 / 90 \mathrm{mmHg})$ and during 24-hour $\mathrm{ABPM}(\leq 130 / 80 \mathrm{mmHg})$ while sustained hypertension is defined by increased pressures in clinic $>140 / 90 \mathrm{mmHg})$ as well as during $\mathrm{ABPM}(\geq 130 / 80 \mathrm{mmHg})$.

$15-30 \%$ of individuals have high clinic blood pressures designated as having White coat hypertension. ${ }^{7}$ It is characterised by high blood pressure $(>140 / 90 \mathrm{mmHg})$ in clinic on minimum three occasions and normal blood pressure $(\leq 130 / 80 \mathrm{mmHg})$ in $\mathrm{ABPM} /$ home monitoring on minimum two occasions without any evidence of target organ damage. ${ }^{8}$ When the differences in systolic and diastolic blood pressures are more than 20 and $10 \mathrm{mmHg}$ respectively, it is considered significant.

White coat hypertension has no specific clinical features and mostly likely occurs in elderly, women, pregnant women, patients diagnosed as stage I hypertension after clinic readings, non-smokers and those without target organ damage. ${ }^{9}$ A study has shown that cardiovascular risk in white coat hypertension is intermediate between normotensive and hypertensive patients, but still closer to normotensive ones..$^{10}$ Another study has shown that frequency of poor cardiovascular events in untreated white coat hypertension individuals is no different than that observed in normotensives. ${ }^{11} \mathrm{ABPM}$ should be performed every year in individuals with white coat hypertension as they have higher probability of developing persistent hypertension. ${ }^{12}$

Prevalence of masked hypertension (white coat normotension) is around $10-40 \%$ in patients not receiving any antihypertensive drugs. It is characterised by clinic blood pressure $(<140 / 90 \mathrm{mmHg})$ and abnormal ABPM readings $(\geq 130 / 80 \mathrm{mmHg}){ }^{6}$ It is commonly seen in males, smokers and those with high body-mass index. It has higher risk of cardiovascular morbidity and mortality than sustained hypertension or white coat hypertension but as clinic blood pressure is normal, this risk may be underrated. ${ }^{10}$ Higher mortality and morbidity may be due to late detection of masked hypertension leading to increased target organ damage. An association has been reported between masked hypertension and risk of structural changes in left ventricle in a meta-analysis of twelve studies. ${ }^{10}$ Therefore antihypertensive therapy seems rational choice for patients with masked hypertension.

\section{ABPM AND DIURNAL BLOOD PRESSURE VARIATION}

It is most reliable method to monitor blood pressure during sleep and its behaviour in 24 hour period during daytime and night. Non-dippers have enhanced risk of cerebrovascular accidents, higher intima-media thickness and target organ damage. ${ }^{13}$ As a result, ABPM has now moved nocturnal BP to centre stage. Another study showed higher mortality rate in those with non-dipping and reverse dipping pattern in comparison to dippers and extreme dippers. ${ }^{14}$ But it was also associated with other risk factors like age, smoking, diabetes mellitus, chronic kidney disease, congestive cardiac failure and coronary artery disease. ${ }^{15}$ Mechanisms involved in night time hypertension and increased mortality have not been elucidated but decreased baroreceptor sensitivity, enhanced sympathetic tone, autonomic dysfunction, sleep apnea, insulin resistance, endothelial dysfunction and increased nocturnal sodium excretion may all be involved. ${ }^{16}$

Sleep-trough morning blood pressure surge (early morning blood pressure - least night blood pressure) has strong association with increased incidence of cerebrovascular disease in untreated hypertension and this surge was found to be significantly more in Japanese population as compared to Europeans independent of age, 24-hour blood pressure and least night-time blood pressure. ${ }^{16}$ Thus morning blood pressure surge is an important parameter to be measured in Asian population using ABPM to prevent cerebrovascular accidents and their recurrence. ${ }^{17}$

\section{BLOOD PRESSURE VARIABILITY}

Till now there was complete reliance on mean measure of ambulatory blood pressure - average of all ambulatory blood pressure measurements over a given time period but there is considerable variability in beat-to-beat blood pressure, so now concept of average real variability (ARV) has come up. Average real variability is shown to be associated with carotid artery damage, left ventricular hypertrophy, cardiovascular events and cardiovascular and all-cause mortality. ${ }^{18-21}$ Average real variability in elderly is associated with cognitive dysfunction and quality of life. ${ }^{22}$ Average real variability is shown to be more in presence of chronic diseases e.g. diabetes mellitus and neurodegenerative diseases. But it is not clear whether 
increased average real variability is cause for progression of disease or a by-product of disease. Efficacy of calcium channel blockers has been found to be maximum in reducing variability in a systematic review and meta-analysis whereas angiotensin receptor blockers and B-blockers were not found to be effective. ${ }^{23}$ One more trial has shown combination of diuretics and calcium channel blockers to be especially potent for lowering short-term variability. ${ }^{24}$

\section{NOCTURNAL HYPERTENSION}

Due to body's circadian rhythm, there is blood pressure variability during day and night. There is a dip in blood pressure which starts late evening and it reaches its trough around mid-night and rises again just after awakening in the morning. ${ }^{25}$ Nocturnal hypertension is blood pressure $\geq$ $120 / 70 \mathrm{mmHg}$ during night and can only be detected by ABPM. It is due to altered circadian rhythm which depends on interaction between sympathetic nervous system and renin-angiotensin-aldosterone system. Nocturnal hypertension is commonly reported in individuals with non-dipping and reverse dipping/riser blood pressure patterns.

Nocturnal hypertension is closely associated with diabetes mellitus, major depression and generalised anxiety disorder which are accompanied by enhanced sympathetic tone during sleep. ${ }^{26,27}$ Bankir et al. have proposed pressure natriuresis hypothesis which suggests that nocturnal rise in blood pressure occurs to cause compensatory increase in sodium excretion so that sodium balance is maintained in body. This can also be explained as that non-dipping pattern of blood pressure results due to decreased sodium excretion by kidney during daytime either due to fall in glomerular filtration rate or increased sodium reabsorption during daytime. ${ }^{28}$

Less melatonin secretion has also been implicated in causing nocturnal hypertension leading to altered blood pressure rhythmicity. Non-dippers have been documented to have less nocturnal melatonin surge. ${ }^{29}$ In addition to this, medications used to treat hypertension have shorter half lives and duration of action and fail to control blood pressure during 24 hours when taken in morning.

\section{NON-DIPPING PATTERN}

Non-dippers require more drugs for optimal control of blood pressure and it can be concluded that non-dipping pattern may represent individuals with severe hypertension. Close association of non-dipping pattern with target organ damage, risk of future cerebrovascular events e.g. intracerebral hemorrhage and secondary hypertension is being seen. Non-dippers also have high prevalence of left ventricular hypertrophy, increased carotid intima-media thickness, microalbuminuria and insulin resistance. ${ }^{30}$ Pulse wave velocity is also high suggesting increased arterial stiffness in non-dippers. ${ }^{31} \mathrm{C}$-reactive protein levels and red cell distribution width are found to be high in non-dippers and these parameters may portend poor cardiovascular outcomes. $^{32}$ Ohasama study has shown an average $20 \%$ increased risk of cardiovascular mortality with each $5 \%$ decrement in night time blood pressure dipping. ${ }^{33}$ Chronotherapy is emerging as new therapeutic option used for managing nocturnal hypertension where one or more drugs is taken in evening or night to obtain normal dipping in accordance with normal circadian rhythm. HOPE study has shown that by night time administration of Ramipril, there was risk reduction of around $22 \%$ in cardiovascular mortality, myocardial infarction and cerebrovascular disease. ${ }^{34}$ Morning versus night dosing of antihypertensive drugs was compared in MAPEC study and it showed that giving drugs at night achieved better overall blood pressure control and there was significantly lower risk of cardiovascular events. ${ }^{35}$ Prevalence of non-dipping also reduced markedly with taking anti-hypertensives at night. Chronotherapy is also found to be beneficial in treating resistant hypertension.

\section{REVERSE/INVERTED DIPPING/RISERS}

It is characterised by unchanged or even higher night blood pressure representing extreme alteration in circadian blood pressure rhythm, This pattern is commonly seen in secondary hypertension, refractory essential hypertension, pre-eclampsia, chronic kidney disease, sleep apnea syndrome and autonomic dysfunction. ${ }^{36}$ Increased sympathetic activity and impaired renal sodium excreting capacity are incriminated in pathogenesis of reverse dipping pattern. Reverse dippers have been shown to have increased prevalence of carotid intima-media thickness, left ventricular mass and microalbuminuria. ${ }^{36}$ Reverse dipping pattern is also associated with lacunar infarcts, spontaneous intracerebral haemorrhage and cardiac failure with preserved systolic function. All cause mortality is found to be two-times more in patients with reverse dipping pattern as compared to dippers and this is attributed to cardiovascular causes. Chronotherapy plays an important role in attaining normal circadian rhythm in reverse dippers also. ${ }^{36}$ Hermida et al. compared effects of Valsartan $(160 \mathrm{mg} /$ day $)$ taken either early morning or at bedtime and it was observed that $75 \%$ of patients administered night time therapy became dippers as compared to $24 \%$ administered morning dose. ${ }^{37} \mathrm{~A}$ Japanese trial evaluated effects of night time Cilnidipine administration and found that blood pressure reduction was more marked 
when drug was given at night-time than in morning in reverse dippers. Drug effect was similar whether given at bedtime or morning in non-dippers and drug was much effective when given in daytime than bedtime in dippers. ${ }^{38}$ But still data regarding this therapeutic approach is scanty and requires further trials and analysis to comment upon beneficial outcomes of this night-time treatment approach.

\section{CONCLUSION}

Non-clinic blood pressure monitoring is an essential method for effective diagnosis, evaluation and devising treatment strategies for hypertension. This method predicts cardiovascular risk associated with hypertension more accurately than clinic blood pressure monitoring. Both home blood pressure and ambulatory blood pressure monitoring include a number of readings making them more reliable and can identify individuals having whitecoat hypertension and masked hypertension. ABPM especially can monitor night-time blood pressure providing information about dipping status and can diagnose patients with non-dipping and reverse dipping pattern. Such patients can be treated with anti-hypertensive chronotherapy to achieve normal blood pressure circadian rhythm and reduce cardiovascular risk associated with these blood pressure patterns. Though the data regarding role of non-clinic blood pressure measurements in guiding treatment is still debatable, but the result of observational studies still favour their preferential use in routine practice.

\section{REFERENCES}

1. Ayman $D$ and Goldshine AD. Blood pressure determinations by patients with essential hypertension. American Journal of Medical Sciences 1940; 200: 465- 474.

2. Gomes MA, Pierin AM, Segre CA and Mion Junior D. Home blood pressure measurement and ambulatory blood pressure measurement versus office blood pressure measurement. Arquivos Brasileiros de Cardiologia 1998; 71(4): 581-585.

3. Parati G, Stergiou GS, Asmar R, Bilo G, de Leeuw P, Imai Y, et al. European Society of Hypertension Working Group on Blood Pressure Monitoring: European Society of Hypertension practice guidelines for home blood pressure monitoring. Journal of Human Hypertension 2010; 24(12):779-785.

4. Peixoto Aldo J. Practical aspects of home and ambulatory blood pressure monitoring. Houston Methodist Debakey Cardiovascular Journal 2015; 11(4):214-218.

5. Parati G, Stergiou G, O'Brien E, Asmar R, Beilin L, Bilo G, et al. Hypertension Working Group on Blood Pressure Monitoring and Cardiovascular Variability: European Society of Hypertension practice guidelines for ambulatory blood pressure monitoring. Journal of Hypertension 2014; 32(7):1359-1366.

6. Nobre F, Mion JuniorD and Mota Gomes MA. V Brazilian Guidelines for Ambulatory Blood Pressure Monitoring and III Brazilian Guidelines for Home Blood Pressure Monitoring. Arquivos Brasileiros de Cardiologia 2011; 97(3 supl 3):1-24.

7. O'Brien E, Parati G, Stergiou G, Asmar R, Beilin L, Bilo G, et al.
European Society of Hypertension Working Group on Blood Pressure Monitoring: European Society of Hypertension position paper on ambulatory blood pressure monitoring. Journal of Hypertension 2013; 31(9):1731 1768.

8. Centers for Medicare and Medicaid Services Baltimore: National Coverage Determination (NCD) for Ambulatory Blood Pressure Monitoring [Online] 2003 Jul 1 [cited 2015 Sep 1]. Available fro $\mathrm{m}$ :

URL:https: // www.cms.gov/medicare- coveragedatabase.

9. Franklin SS, Thijs L, Hansen TW, O'Brien E and Staessen JA. White-coat hypertension: new insights from recent studies. Hypertension 2013; 62(6):982-987.

10. Pierdomenico SD and Cuccurullo F. Prognostic value of whitecoat and masked hypertension diagnosed by ambulatory monitoring in initially untreated subjects: an updated metaanalysis. American Journal of Hypertension 2011; 24(1):52-58.

11. Franklin SS, Thijs L, Hansen TW, Li Y, Boggia J, Kikyua M, et al. International Database on Ambulatory Blood Pressure in Relation to Cardiovascular Outcomes investigators. Significance of white-coat hypertension in older persons with isolated systolic hypertension: a meta analysis using the International Database on Ambulatory Blood Pressure Monitoring in Relation to Cardiovascular Outcomes Population. Hypertension 2012; 59(3):564-571.

12. Hoshide $\mathrm{S}$, Kario $\mathrm{K}$, Hoshide $\mathrm{Y}$, Umeda $\mathrm{Y}$, Hashimoto $\mathrm{T}$, Kunii $\mathrm{O}$, et al. Associations between nondipping of nocturnal blood pressure decrease and cardiovascular target organ damage in strictly selected community-dwelling normotensives. American Journal of Hypertension 2003; 16:434-438.

13. Ben-Dov IZ, Kark JD, Bem-Ishay D, Mekler J, Bem-Arie L and Bursztyn M. Predictors of all-cause mortality in clinical ambulatory monitoring: unique aspects of blood pressure during sleep. Hypertension 2007; 49(6):1235-1241.

14. Brotman DJ, Davidson MB, Boumitri M and Vidt DG. Impaired diurnal blood pressure variation and all cause mortality. American Journal of Hypertension 2008; 21(1):92-97.

15. Ortega KC, da Silva GV and Mion D Jr. Nocturnal blood pressure fall changes in correlation with urinary sodium excretion. Hypertension 2008; 52(2):e10.

16. Kario K, Pickering TG, Umeda Y, Hoshide S, Hoshide Y, Morinari M, et al. Morning surge in blood pressure as a predictor of silent and clinical cerebrovascular disease in elderly hypertensives: $\mathrm{A}$ prospective study Circulation 2003; 107:1401-1406.

17. Hoshide S, Kario K, de la Sierra A, Bilo G, Schillaci G, Banegas JR, et al. Ethnic differences in the degree of morning blood pressure surge and in its determinants between Japanese and European hypertensive subjects: Data from the ARTEMIS study. Hypertension 2015; 66:750-756.

18. Mancia G, Parati G, Hennig M, Flatau B, Omboni S, Glavina F, et al. Relation between blood pressure variability and carotid artery damage in hypertension: baseline data from the European Lacidipine Study on Atherosclerosis (ELSA), Journal of Hypertension 2001; 19:1981-1989.

19. Veerman DP, de Blok K and van Montfrans GA. Relationship of steady state and ambulatory blood pressure variability to left ventricular mass and urinary albumin excretion in essential hypertension. American Journal of Hypertension 1996; 9:455-460.

20. Björklund $K$, Lind $L$, Zethelius $B$, Berglund $L$ and Lithell $H$. Prognostic significance of 24-h ambulatory blood pressure characteristics for cardiovascular morbidity in a population of elderly men. Journal of Hypertension 2004; 22:1691-1697.

21. Kikuya M, Hozawa A, Ohokubo T, Tsuji I, Michimata M, Matsubara M, et al. Prognostic significance of blood pressure and heart rate variabilities the Ohasama study. Hypertension 2000; 36:901-906. 
22. Sakakura K, Ishikawa J, Okuno M, Shimada K and Kario K. Exaggerated ambulatory blood pressure variability is associated with cognitive dysfunction in the very elderly and quality of life in the younger elderly. American Journal of Hypertension 2007; 20:720-727.

23. Webb AJ, Fischer U, Mehta $Z$ and Rothwell PM. Effects of antihypertensive-drug class on inter-individual variation in blood pressure and risk of stroke: a systematic review and metaanalysis. The Lancet 2010; 375:906-915.

24. Levi-Marpillat N, Macquin-Mavier I, Tropeano A, Parati G and Maison P. Antihypertensive drug classes have different effects on short-term blood pressure variability in essential hypertension. Hypertension Research 2014; 37:1-6.

25. Pickering TG. The clinical significance of diurnal blood pressure variations: Dippers and nondippers. Circulation. 1990; 81(2):700-702.

26. Rossen NB, Knudsen ST, Fleischer J, Hvas AM, Ebbehøj E, Poulsen PL, et al. Targeting nocturnal hypertension in type 2 diabetes mellitus. Hypertension 2014; 64: 1080-1087.

27. Kayano H, Koba S, Matsui T, Fukuoka H, Toshida T, Sakai T, et al. Anxiety disorder is associated with nocturnal and early morning hypertension with or without morning surge. Circulation Journal 2012; 76:1670-1677.

28. Bankir L, Bochud M, Maillard M, Bovet P, Gabriel A and Burnier M. Nighttime blood pressure and nocturnal dipping are associated with daytime urinary sodium excretion in African Subjects. Hyาpertension 2008; 51: 891-898.

29. Pechanova $O$, Paulis $L$ and Simko F. Peripheral and central effects of melatonin on blood pressure regulation. International Journal of Molecular Sciences 2014; 15: 17920-17937.

30. Birkenhäger $A M$ and van den Meiracker $A H$. Causes and consequences of a non-dipping blood pressure profile. The Netherlands Journal of Medicine 2007; 65(4):127-131.
31. Cicek Y, Durakoglugil ME, Kocaman SA, Cetin M, Erdogan T, Dogan S, et al. Non-dipping pattern in untreated hypertensive patients is related to increased pulse wave velocity independent of raised nocturnal blood pressure. Blood Pressure 2012; 22(1): 34-38.

32. Gunebakmaz O, Kaya MG, Duran M, Akpek M, Elcik D and Eryol NK. Red blood cell distribution width in 'non dippers' versus 'dippers'. Cardiology. 2012; 123(3):154-159.

33. Ohkubo T, Hozawa A, Yamaguchi J, Kikuya M, Ohmori K, Michimata $\mathrm{M}$, et al. Prognostic significance of the nocturnal decline in blood pressure in individuals with and without high 24 hour blood pressure: the Ohasama study. Journal of Hypertension 2002; 20(11):2183-2189.

34. Sleight P, Yusuf S, Pogue J, Tsuyuki R, Diaz R and Probstfield J, for Heart Outcomes Prevention Evaluation (HOPE) Study. Blood-pressure reduction and cardiovascular risk in HOPE study. The Lancet 2001; 358(9299):2130-2131.

35. Hermida RC, Ayala DE, Mojón A, Fernández JR. Influence of circadian time of hypertension treatment on cardiovascular risk: results of the MAPEC study. Chronobiology International 2010; 27(8):1629-1651.

36. Cesare Cuspidi, Sala C, Tadic M, Gherbesi E, De Giorgi A, Grassi G, et.al. Clinical and prognostic significance of a reverse dipping pattern on ambulatory monitoring: An updated review. The Journal of Clinical Hypertension 2017; 19:713-721.

37. Hermida RC, Calvo C, Ayala DE, Fernández JR, Covelo M, Mojón A, et al. Treatment of non dipper hypertension with bedtime administration of valsartan. Journal of Hypertension 2005;23:1913-1922

38. Kario K, Nariyama J, Kido H, Ando S, Takiuchi S, Eguchi K, et al. Effect of a novel calcium channel blocker on abnormal nocturnal blood pressure in hypertensive patients. The Journal of Clinical Hypertension 2013; 15:465-472.

\footnotetext{
Authors Contribution:

VK-Concept of article, review of literature, manuscript preparation and critical revision of manuscript.
}

Work attributed to: Associate Professor, Department of Medicine,Dr H S Judge Institute Of Dental sciences,Panjab University, Chandigarh.

Orcid ID:

Dr.Vinay Kapur - (D) https://orcid.org/0000-0003-4281-1111

Source of Support: Nil, Conflict of Interest: None. 